Report

\title{
Utilization of professional supportive care services by women with breast cancer
}

Ross E. Gray ${ }^{1}$, Vivek Goel ${ }^{2}$, Margaret I. Fitch ${ }^{1}$, Edmee Franssen ${ }^{3}$, Pamela Chart ${ }^{4}$, Marlene Greenberg ${ }^{4}$, Debra Bakker ${ }^{5}$, Manon Labrecque ${ }^{1}$, Eric Hollowaty ${ }^{6}$, Raylene Godel ${ }^{7}$, and Anne Wray Hampson ${ }^{8}$

${ }^{1}$ Psychosocial \& Behavioural Research Unit, Toronto-Sunnybrook Regional Cancer Centre; ${ }^{2}$ Department of Health Administration, Faculty of Medicine, University of Toronto; ${ }^{3}$ Clinical Trials \& Epidemiology, ${ }^{4}$ Preventive Oncology, Toronto-Sunnybrook Regional Cancer Centre; ${ }^{5}$ Nursing Research, Northeastern Regional Cancer Centre; ${ }^{6}$ Ontario Cancer Registry; ${ }^{7}$ Cancer Care Ontario; ${ }^{8}$ Willow Breast Cancer Support \& Resources, Toronto, Ontario, Canada

Key words: breast cancer, psychosocial, supportive care, utilization

\section{Summary}

This paper reports on the results of a survey of utilization of professional supportive care services by women with breast cancer, and on patterns of differential service utilization by sub-groups of patients. Study participants were women with invasive breast cancer diagnosed 23-36 months prior to contact about the study, and randomly selected from the Ontario Cancer Registry. From among 1,119 eligible women sent survey questionnaires, 731 returned completed questionnaires $(65 \%)$. A total of $31 \%$ of respondents reported accessing one or more of the following professionals: social worker, psychologist, psychiatrist, dietitian, physiotherapist. Among those who responded to a question about whether they would have liked specific services, 34\% reported that there was at least one professional supportive care service they would have liked to use, but were unable to access. Factors shown to be related to greater utilization of services included: younger age, higher household income, employed or student status, private health insurance coverage, and having received chemotherapy. Overall, there was a surprisingly low utilization of professional specialized supportive care services among women with breast cancer. Policy implications include finding strategies to better inform cancer patients about existing services, and ensuring that a core set of services are available to all patients.

\section{Introduction}

Supportive cancer care is an umbrella term referring to interventions which supplement medical attempts to control or eradicate disease. It has been defined as, "The provision of the necessary services for those living with or affected by cancer to meet their physical, psychosocial, informational and spiritual needs during the diagnostic, treatment, and follow-up phases, encompassing issues of survivorship, palliation and bereavement" [1]. Supportive care can arguably refer to a very broad set of services - including professionally offered services and lay or self-help services. Some supportive care services exist as part of the formal cancer care system, while others are available exclusively in community settings where they are accessed through formal or informal networks or organizations. In this paper we are concerned with professional services that specialize in supportive (as opposed to medical) care and are considered part of the formal cancer care system.

Most of the literature related to supportive care in cancer has focused either on the identification of problems that might indicate patient needs for assistance, [2-5] or on the effectiveness of specific interventions for alleviating problems within the supportive care domain [6-8]. There has been relatively little attention paid to the utilization of supportive care services, 
especially as contrasted to health systems research into utilization of medical interventions for cancer [9].

One British study reported on the results of surveys about support services sent to administrators of Health districts [10]. A similar US study reported on survey results obtained from medical and nursing directors of National Cancer Institute comprehensive cancer centres [11]. In both of these studies, considerable variety and fragmentation of service was reported. Important limitations of these studies had to do with reliance on administrative or professional definitions of service availability without consideration for individual patient perspectives.

Results of studies of cancer patient needs in four Canadian provinces indicated that health professionals other than physicians were rarely noted by patients as being sources of information and support - suggesting a low level of overall utilization of specialized supportive care services [12].

The lack of adequate data on utilization of supportive care services has had policy implications for the management of cancer care in western countries. Health system administrators have had to make decisions about the priorities of services, staffing quotas, and models of service delivery outside of the context of meaningful information. More pointedly, the lack of data about expressed needs and utilization of services has made it easier for managers to reduce or eliminate supportive care services.

In this paper, our focus is on the utilization of supportive care services among breast cancer patients in Ontario, Canada. The importance of such services for breast cancer patients has been evidenced by their advocacy efforts to ensure that supportive care needs are met [13].

\section{Methods}

Participants for this study were women with histologically confirmed invasive breast cancer diagnosed 23-36 months prior to contact about the study, randomly selected from the Ontario Cancer Registry (94\% of cases in the Registry are histologically confirmed). The physician listed first on the pathology report (most often a surgeon) was contacted for permission to approach individual patients. Physicians were asked to indicate if there was a more appropriate colleague to approach about obtaining permission.
In those cases where another physician was indicated as more appropriate, or where we were unable to contact the first physician, we pursued alternate sources for physician approval (e.g. family physician). Breast cancer patients for whom a physician had approved contact were mailed a survey questionnaire, covering letter, and stamped, self-addressed return envelope. Three weeks later, a reminder card was mailed. Finally, after another three weeks, an additional letter, survey questionnaire, and return envelope were sent to those who had not yet returned the survey questionnaire.

The survey questionnaire provided a list of specialized supportive care services. Respondents were instructed to indicate which of these services they had used to meet supportive care needs related to their breast cancer since they were diagnosed. If services were not utilized, women were asked if they would have liked to use them. They were also asked whether any family members had utilized supportive care services to help deal with the respondent's breast cancer. Items related to social and demographic characteristics were included in the survey questionnaire, as were items related to illness and treatment information.

Professional supportive care services reported on in this paper are those supported (paid for) by the Ontario health care system. These services (social workers, psychologists, psychiatrists, dietitians, physiotherapists) are provided in cancer treatment facilities (although not all services are necessary available within each facility), where patients are able to see professionals at no cost (at least for a number of visits).

The survey questionnaire items were constructed through the input of multiple experts, including women with breast cancer, supportive care personnel, physicians, and nurses. Two focus groups were held with breast cancer patients. Members of the focus groups completed draft versions of the questionnaire and provided feedback about possible areas for improvement.

Descriptive statistics (frequencies, means, modes) were employed in analyzing the data. In addition, logistic regression analysis was conducted to assess the relationship of individual demographic and illness/treatment factors to utilization of professional supportive care services. Then, in an attempt to assess the influence of the same demographic and illness/treatment factors in the context of the influences of other factors, stepwise logistic regression analyses were conducted. 


\section{Results}

Ontario physicians were sent forms requesting permission to approach 2,902 breast cancer patients diagnosed in 1995 and selected randomly from the Ontario Cancer Registry. Physicians returned completed forms for 1,659 patients (57\%). Approval for contact was given for 1,197 of the 1,659 women for whom forms were returned $(72 \%)$. When reasons were provided for not approving contact, these included: (1) not knowing who the doctor currently in charge of the patient was $(N=198)$; (2) patient died $(N=86)$; (3) physician didn't want patient to participate $(N=44)$; (4) physician retired, moved or declined to participate $(N=26)$.

Of the 1,197 questionnaires mailed to women in early 1998, 58 were returned incomplete due to wrong addresses, and an additional 20 were returned because the women had died. Of the remaining 1,119 eligible women, 731 completed and returned questionnaires $(65 \%)$.

A majority (62.9\%) of women responding to this survey were 60 years old or less (see Table 1). Most $(73 \%)$ were married or living with an intimate partner, and had completed at least a secondary school education $(88 \%)$. Approximately half $(48 \%)$ had a family income of $\$ 40,000$ or more and had health insurance beyond that which was provided by the government $(53 \%)$. English was the first language for $82 \%$ of respondents.

Women for whom physicians gave approval did not differ significantly by age or urban versus rural residence from those whom physicians did not give approval. However, from among women who sent the questionnaire, a higher proportion of those less than 65 years old returned it than did older women $(69 \%$ vs $57 \%)$.

A total of $31 \%$ of respondents had used at least one professional supportive care service. Table 2 shows the proportion of women who utilized each of the specific services. Only $2 \%$ of respondents reported that at least one family member had utilized at least one professional supportive care service to assist them in dealing with the patient's breast cancer. Among those who responded to a question about whether they would have liked specific services, $34 \%$ of respondents reported that there was at least one professional supportive care service they would have liked to use, but were unable to access.

Using logistic regression analyses, we examined demographic and illness/treatment variables to as-
Table 1. Demographic and disease/treatment information and proportion using one or more professional supportive care services

\begin{tabular}{|c|c|c|}
\hline & $\%$ Overall & $\begin{array}{l}\% \text { Using } \\
\text { service(s)-95 CI }\end{array}$ \\
\hline \multicolumn{3}{|l|}{ Age $^{*}$} \\
\hline$<65$ yrs & 72 & $38(34,42)$ \\
\hline $65+\mathrm{yrs}$ & 27 & $13(8,18)$ \\
\hline \multicolumn{3}{|l|}{ Work status* } \\
\hline Working or student & 41 & $41(35-46)$ \\
\hline Other & 59 & $25(21,29)$ \\
\hline \multicolumn{3}{|l|}{ Household income* } \\
\hline$<\$ 40,000$ & 48 & $24(19,29)$ \\
\hline$\$ 40,000+$ & 41 & $39(33,44)$ \\
\hline \multicolumn{3}{|l|}{ Treatments received* } \\
\hline Chemotherapy & 41 & $44(39,50)$ \\
\hline No chemotherapy & 59 & $23(19,27)$ \\
\hline \multicolumn{3}{|c|}{ Health insurance in addition to government-provided* } \\
\hline Yes & 53 & $37(33,42)$ \\
\hline No & 47 & $25(21,30)$ \\
\hline \multicolumn{3}{|l|}{ Marital status } \\
\hline Married/intimate partner & 73 & $33(29,37)$ \\
\hline Other & 26 & $29(22,35)$ \\
\hline \multicolumn{3}{|l|}{ Education } \\
\hline High school or more & 88 & $25(16,34)$ \\
\hline$<$ High school & 12 & $33(29,36)$ \\
\hline \multicolumn{3}{|l|}{ Recurrence or relapse } \\
\hline Yes & 11 & $37(27,48)$ \\
\hline No & 85 & $31(27,34)$ \\
\hline
\end{tabular}

$n=731 ;{ }^{*}=$ Significant at $p<0.05$.

Table 2. Number of respondents reporting utilization of professional supportive care services

\begin{tabular}{llr}
\hline & $N$ & $\%$ \\
\hline Physiotherapist & 103 & 14 \\
Registered dietitian & 93 & 13 \\
Social worker & 79 & 11 \\
Psychologist & 38 & 5 \\
Psychiatrist & 26 & 4 \\
\hline
\end{tabular}

sess their relationship to women's utilization of professional supportive care services. Utilization was related to younger age, working or student status, additional health insurance, higher household income, and receiving chemotherapy treatment. The reporting of one or more professional supportive care services that were wished for but not received was related to younger age, working or stu- 
Table 3. Influence of factors on use of at least one professional supportive care service (Results of logistic regression)

\begin{tabular}{lll}
\hline & Odds ratio & $95 \%$ CI \\
\hline$<65$ years & 3.61 & $2.15-6.06$ \\
Received chemotherapy & 1.90 & $1.31-2.74$ \\
\hline
\end{tabular}

dent status, higher education, and additional health insurance.

To learn which of these factors were independently related to utilization of professional supportive care services, stepwise multiple logistic regression analyses were conducted. All factors with significant univariate associations with utilization were entered into their respective logistic regressions to detect the effect of each variable, while simultaneously controlling for the effects of other variables. Utilization of professional services supported by the cancer system was significantly and independently associated with younger age and receiving chemotherapy (Table 3). Reporting of at least one supportive care service wished for but not received, when analyzed using stepwise multiple logistic regression, was significantly and independently associated with younger age (Odds ratio $=4.45 ; 95 \% \mathrm{CI}=2.26-8.77$ )

\section{Discussion}

It is important to first consider limitations of our study. The sample for this survey study was drawn from the population of Ontario women diagnosed with invasive breast cancer during a one-year period. In comparing the data available on pathology reports (age of patient and geographical location of attending physician), we found no reason to suspect that the women approved for contact by physicians were different as a group from those for whom approval was not granted. However, the women who returned the questionnaire varied from those who did not return the questionnaire. Data derived from the Ontario Cancer Registry showed that respondents were younger than non-respondents. Although other demographic data was not available for the nonrespondents, comparisons of respondents with Statistics Canada reports suggests that there was an over-representation of women who were well educated, had higher than average annual income, and whose first language was English. These findings have implications for the generalizability of our findings, and may suggest that the utilization of supportive care services is actually less than our data reveal, given that it is exactly the younger, more privileged women in our sample who made the most use of services. While differences between the Canadian and US health care systems may mean that the findings of this study are not entirely transferable, cancer treatment facilities in both countries attempt to provide their patients with appropriate access to specialized supportive care services. And in both countries there are formulas that allow at least some portion of the cost of such services to be paid for by sources other than individual patients.

The utilization of professional supportive care services supported by the cancer care system was lower among breast cancer patients than what might be expected or hoped for. When considered in the light of recommendations that, for example, all patients would benefit from nutritional guidance from a registered dietitian, $[14,15]$ or that a high proportion of patients should receive the attention of a social worker, [16] our findings are worrisome.

A complicating factor in understanding the meaning of the finding of low utilization is that availability of professional supportive care services varies across the province. This is by no means a feature exclusive to the Ontario system, as both US and British studies have reported the same type of variability. This means that the level of utilization was likely affected by lack of services in some cases, while in other cases there were issues of access to existing services. Further clarification for why individuals do not receive supportive care services is required. To that end, we are currently investigating barriers to utilization of services through a series of semi-structured interviews with a sub-sample of survey respondents. Findings will be reported in a future publication.

It is now widely acknowledged that cancer is best considered a family illness, because of the profound effects that serious illness has within families [17]. Our finding that only $2 \%$ of women reported any family member using a professional supportive care service suggests that there has been little movement from the idea of the importance of the family towards cancer system action to attend to family needs.

Younger breast cancer patients were more likely than older women to use specialized supportive care services. This finding is consistent with those in the screening and treatment domains, where study results led to conclusions that older women were under-serviced, and that greater efforts were required 
to ensure their access to services. It is less clear what conclusions should be arrived at for our study. Younger women may have legitimately greater needs for supportive care services due to multiple demands of children, work, etc. Yet, did their greater utilization of services only reflect their greater needs, or there are also generational factors that make it more difficult for older women to ask for and seek out services that they might benefit from? We suspect that our findings reflect both aspects.

Breast cancer patients who were employed, had higher incomes, and subscribed to private health care insurance plans to supplement governmental health insurance, made greater use of supportive care services. These findings reflect the reality of Canadian health care, in which supportive care falls at least partially outside of the umbrella of government-provided health insurance. Given this situation, those with more resources at their disposal have greater choice about the services they can access. But these findings also likely reflect the social phenomenon that the more people have (both in terms of resources and education), the more they come to expect, and the more motivated they may thus be to seek out services to meet perceived needs.

Utilization of professional supportive care services was influenced by the treatments received by breast cancer patients, with those who underwent the rigors of chemotherapy and/or stem cell transplants more likely to require supportive care. This finding was expected, and suggests that more than just the politics of socioeconomic advantage are at play in determining who gets help. Clearly, those who are most sick require specialized supportive care services, and, according to our data, they are more likely to be receiving them.

Policy implications from this study relate to the surprisingly low utilization of professional supportive care services overall. One strategy to address this situation is to better inform patients about existing services. To provide such information implies that medical and nursing practitioners need to be knowledgeable about supportive care services. Educational initiatives may be warranted with these groups. The assessment of supportive care needs should be part of every visit to cancer treatment centers, and practitioners should be engaged in ongoing dialogues with patients about what types of additional assistance might be helpful. One important aspect of such conversations is to help patients understand that needing help beyond the purely medical is in no way a failure, and that many patients will benefit from assistance at some point along the illness continuum.

While information strategies are of critical importance to ensuring appropriate utilization of supportive care services, they are only relevant where services are available. As noted above, all data gathered to date suggest that availability of services around North America, including Ontario, are remarkably varied. But this isn't good enough. Policy makers have a responsibility to ensure that a core set of supportive care services are available to all cancer patients.

\section{Acknowledgements}

This research was supported by the Canadian Breast Cancer Research Initiative, and was conducted with the assistance of Cancer Care Ontario's cancer registry.

\section{References}

1. Fitch M: Providing Supportive Care for Individuals Living with Cancer. Toronto, Ontario Cancer Treatment and Research Foundation, 1994

2. Whelan TJ, Mohide EA, Willan AR, et al.: The supportive care needs of newly diagnosed cancer patients attending a regional cancer center. Cancer 15: 1518-1524, 1997

3. Grande GE, Todd CJ, Barclay SIG: Support needs in the last year of life: Patient and carer dilemmas. Palliat Med 11: 202208, 1997

4. Ferrell BR, Grant MM, Funk BM, et al.: Quality of life in breast cancer survivors: Implications for developing support services. Oncol Nurs Forum 25: 887-895, 1998

5. Massie MJ, Holland JC: Overview of normal reactions and prevalence of psychiatric disorders. In: Holland JC, Rowland JH (eds) Handbook of Psychooncology. Oxford Press, New York, 1990, pp 273-282

6. Massie MJ, Holland JC, Straker N: Psychotherapeutic interventions. In: Holland JC, Rowland JH (eds): Handbook of Psychooncology. Oxford Press, New York, 1990, pp 455-469

7. Mastrovito R: Behavioral techniques: Progressive, relaxation and self-regulatory techniques. In: Holland JC, Rowland JH (eds): Handbook of Psychooncology. Oxford Press, New York, 1990, pp 492-501

8. Avants SK, Margolin A, Singer JL: Psychological interventions and research in the oncology setting: An integrative approach. Psychotherapy 30: 1-10, 1993

9. Goel V: Health services research in breast cancer: Background paper for a Canadian breast cancer research initiative workshop. Cancer Prev Control 2: 173-178, 1998

10. Addington-Hall JM, Weir MW, Zollman C, et al.: A national survey of the provision of support services for people with cancer. BMJ 304: 1649-1650, 1993

11. Coluzzi PH, Grant M, Doroshow JH, et al.: Survey of the provision of supportive care services at National Cancer Institute-designated centers. J Clin Oncol 13: 756-764, 1995 
12. Final Report on the Needs of People Living with Cancer Across Canada. Toronto, Canadian Cancer Society, 1992

13. Report on the National Forum on Breast Cancer. Ottawa, Ontario, Health Canada, 1994

14. Rohan TE, Hiller JE, McMichael AJ: Dietary factors and survival from breast cancer. Nutr Cancer 20: 167-177, 1993

15. Ireton-Jones CS, Garritson B, Kitchens L: Nutrition intervention in cancer patients: Does the registered dietitian make a difference? Top Clin Nutr 10: 42-48, 1995
16. Christ G: Social work in oncology. In: Holland JC, Rowland JH (eds) Handbook of Psychooncology. Oxford Press, New York, 1990, pp 670-677

17. Northouse LL: Family issues in cancer care. Adv. Psychosom. Med. 18: 82-101, 1988

Address for offprints and correspondence: Ross E. Gray, Psychosocial \& Behavioural Research Unit, Toronto-Sunnybrook Regional Cancer Centre, 2075 Bayview Avenue, Canada M4N 3M5; Tel: 416-480-5866; Fax: 416-480-5144; E-mail: ross.gray@tsrcc.on.ca 